\title{
Role of the forkhead protein FoxO1 in $\beta$ cell compensation to insulin resistance
}

\author{
Haruka Okamoto, ${ }^{1,2}$ Marta Letizia Hribal, ${ }^{1}$ Hua V. Lin, ${ }^{1}$ \\ William R. Bennett, ${ }^{3}$ Andrew Ward, ${ }^{3}$ and Domenico Accili 1
}

\begin{abstract}
1Department of Medicine and 2Institute of Human Nutrition, College of Physicians and Surgeons of Columbia University, New York, New York, USA. ${ }^{3}$ Department of Biology \& Biochemistry, University of Bath, Bath, United Kingdom.
\end{abstract}

\begin{abstract}
Diabetes is associated with defective $\beta$ cell function and altered $\beta$ cell mass. The mechanisms regulating $\beta$ cell mass and its adaptation to insulin resistance are unknown. It is unclear whether compensatory $\beta$ cell hyperplasia is achieved via proliferation of existing $\beta$ cells or neogenesis from progenitor cells embedded in duct epithelia. We have used transgenic mice expressing a mutant form of the forkhead-O1 transcription factor (FoxO1) in both pancreatic ductal and endocrine $\beta$ cells to assess the contribution of these 2 compartments to islet expansion. We show that the mutant FoxO1 transgene prevents $\beta$ cell replication in 2 models of $\beta$ cell hyperplasia, 1 due to peripheral insulin resistance (Insulin receptor transgenic knockouts) and 1 due to ectopic local expression of IGF2 (Elastase-IGF2 transgenics), without affecting insulin secretion. In contrast, we failed to detect a specific effect of the FoxO1 transgene on the number of periductal $\beta$ cells. We propose that $\beta$ cell compensation to insulin resistance is a proliferative response of existing $\beta$ cells to growth factor signaling and requires FoxO1 nuclear exclusion.
\end{abstract}

\section{Introduction}

The mechanisms regulating $\beta$ cell compensation to insulin resistance and their eventual failure in type 2 diabetes are incompletely understood (1). $\beta$ cells arise from neurogenin 3 -expressing endocrine progenitor cells during embryogenesis $(2,3)$ and are renewed by replication during adult life (4). Uncertainty persists as to the role of neogenesis, i.e., the generation of endocrine cells from undifferentiated or partially differentiated precursors, in the maintenance of $\beta$ cell mass in the adult pancreas (5). While the presence of hormone-positive cells within or near pancreatic ducts has been taken as evidence that duct epithelia are sites of $\beta$ cell neogenesis (6), this conclusion is not supported by lineage-tracing studies (7). It is unclear whether proliferation or neogenesis governs $\beta$ cell hyperplasia in insulin-resistant states and what drives expansion of $\beta$ cell mass. Many growth factors can increase $\beta$ cell proliferation (8-10), but there is no evidence that the metabolic syndrome increases systemic growth factor levels. Similarly, although both glucose (11) and free fatty acids (12) can promote $\beta$ cell proliferation, the increase of $\beta$ cell mass in mouse models of insulin resistance precedes the onset of these metabolic changes.

Studies of mice with targeted gene mutations indicate that the insulin signaling pathway regulates several aspects of pancreatic $\beta$ cell function, including hormone secretion and proliferation (13). For example, mice lacking insulin receptor (Insr) (14), Igf1 receptor $(15,16)$, or Insr substrate 1 (Irs1) (17) show impaired insulin secretion while mice nullizygous for Insr substrate 2 (Irs2) develop $\beta$ cell failure due to decreased proliferation/increased apoptosis $(18,19)$. Ablation of the regulatory subunit (p85) of phosphoinositol-3 kinase results in constitutive insulin secretion (20) while overexpression of constitutively active Akt causes $\beta$ cell

Nonstandard abbreviations used: Foxo, forkhead-O transcription factor; Insr, insulin receptor; Irs1, insulin receptor substrate 1.

Conflict of interest: The authors have declared that no conflict of interest exists. Citation for this article: J. Clin. Invest. doi:10.1172/JCI24967. hyperplasia but does not affect basal insulin secretion $(21,22)$. The forkhead-O (FoxO) subfamily of transcription factors plays an important role in insulin action. We have shown that FoxO1 haploinsufficiency reverses $\beta$ cell failure in Irs $2^{-/-}$mice through partial restoration of $\beta$ cell proliferation, positioning FoxO1 as a key distal mediator of Insr signaling in $\beta$ cells (23). The ability of FoxO1 to restore $\beta$ cell mass in Irs $2^{-/-}$mice is associated with increased expression of $\mathrm{Pdx} 1$, a critical regulator of $\beta$ cell survival (24), suggesting that FoxO1 is a repressor of $P d x 1$ expression. We have further shown that transgenic expression of constitutively nuclear FoxO1 in liver causes impaired fasting glucose by increasing expression of glucogenic enzymes, as does combined expression of the transgene in liver and $\beta$ cells (25). The metabolic consequences of expressing the mutant FoxO1 in $\beta$ cells remain poorly defined, as does the role of FoxO1 in $\beta$ cell adaptation to insulin resistance.

In this study, we used transgenic mice expressing a constitutively nuclear FoxO1 (25) to examine the mechanism of $\beta$ cell adaptation to insulin resistance. We summarize the features of the mouse strains at the beginning of Results. Using experimental crosses between FoxO1 transgenic mice (25) and Insr transgenic knockouts (26), we show that constitutive nuclear expression of FoxO1 in $\beta$ cells prevents $\beta$ cell hyperplasia and accelerates the onset of diabetes, despite a compensatory increase in insulin secretion. To rule out that the observed failure of $\beta$ cell proliferation is due to the complex genetic makeup of the FoxO1/Insr double-transgenic mice (26), we also studied the effects of the FoxO1 transgene in a model of "pure" $\beta$ cell hyperplasia, brought about by ectopic expression of IGF2 (referred to as El-IGF2 mice). Similar to FoxO1/Insr mice, FoxO1/El-IGF2 double-transgenic mice showed impaired expansion of $\beta$ cell mass. When viewed in the context of previous work with FoxO1 haploinsufficient mice (23), the data indicate that FoxO1 signaling is an integral part of the $\beta$ cell response to insulin resistance. The latter, in turn, appears to consist primarily of proliferation of existing $\beta$ cells. 
Table 1

Experimental crosses

\begin{tabular}{|c|c|c|c|c|c|}
\hline Strain & Derivation & Metabolic phenotype & Sites of insulin resistance & Expected outcome & $\beta$ cell mass ${ }^{A}$ \\
\hline L1 & $\begin{array}{l}\text { Insr }{ }^{-} \text {- rescued by Ttr-Insr } \\
\text { transgene expressed in } \\
\text { liver, brain, and } \beta \text { cells }\end{array}$ & $\begin{array}{l}\text { Hyperinsulinemia, } \\
\text { euglycemia }\end{array}$ & Muscle, fat & $\mathrm{N} / \mathrm{A}$ & $\times 2-3$ \\
\hline 307 & $\begin{array}{l}\text { Fox } 01^{\mathrm{S} 253 \mathrm{~A}} \text { transgene } \\
\text { expressed in liver }\end{array}$ & $\begin{array}{l}\text { Impaired fasting } \\
\text { glucose }\end{array}$ & Liver & $\mathrm{N} / \mathrm{A}$ & Normal \\
\hline 305 & $\begin{array}{l}\text { Fox01 }{ }^{\mathrm{S} 253 \mathrm{~A}} \text { transgene } \\
\text { expressed in } \beta \text { cells, liver }\end{array}$ & $\begin{array}{l}\text { Impaired fasting } \\
\text { glucose }\end{array}$ & $\beta$ cells (?), liver & $\mathrm{N} / \mathrm{A}$ & Normal \\
\hline $\mathrm{L} 1 \times 307$ & This study & This study & Muscle, fat, liver & $\begin{array}{c}\text { Diabetes with } \\
\beta \text { cell hyperplasia }\end{array}$ & $\times 2-3$ \\
\hline $\mathrm{L} 1 \times 305$ & This study & This study & Muscle, fat, liver, $\beta$ cells & Diabetes with $\beta$ cell failure & $\times 1.6$ \\
\hline IGF2 & This study & This study & Not present & $\beta$ cell hyperplasia & $\times 3$ \\
\hline IGF2 × 305 & This study & $\begin{array}{l}\text { Hyperinsulinemia, } \\
\text { euglycemia }\end{array}$ & None & Reduced $\beta$ cell mass & $\times 0.6$ \\
\hline
\end{tabular}

L1 mice are Insr transgenic knockouts in which Insr expression is limited to liver, brain, and $\beta$ cells. They are insulin resistant and nondiabetic. The 307 mice express a constitutively active FoxO1 transgene in liver while 305 mice express a constitutively active FoxO1 transgene in $\beta$ cells and liver. They have mild fasting hyperglycemia and modest hyperinsulinemia. When L1 and 307 mice are intercrossed, the expectation is that the FoxO1 transgene will impair hepatic insulin action and cause further insulin resistance and diabetes. When L1 and 305 mice are intercrossed, the expectation is that the FoxO1 transgene will impair both hepatic insulin action and $\beta$ cell compensation. When IGF2 mice and 305 mice are intercrossed, the expectation is that $\beta$ cell mass will decrease but, because there is no additional metabolic impairment, mice will be euglycemic. AFold increase in mg.

\section{Results}

To study the mechanism of compensatory $\beta$ cell mass expansion in insulin resistance, we generated diabetic mice with mutations affecting insulin signaling and $\beta$ cell replication. In the first experimental cross, we intercrossed Ins $r$ transgenic knockout mice, in which Insr is expressed only in liver, brain, and $\beta$ cells (henceforth, L1) (26), with transgenic mice expressing a constitutively nuclear FoxO1 in liver (henceforth, 307) (25). The mutation replaces a key phosphorylation site (Ser253Ala), thereby preventing phosphorylation-dependent nuclear exclusion (25). The expectation was that the FoxO1 transgene would impair insulin action in liver and cause a compensatory increase in $\beta$ cell mass (Table 1 ).

In the second experimental cross, we intercrossed L1 mice with transgenics expressing constitutively nuclear FoxO1 in $\beta$ cells, pancreatic ducts, and liver (henceforth, 305). The expectation here was that, in addition to impairing liver insulin action, expression of the mutant FoxO1 in $\beta$ cells would curtail expansion of $\beta$ cell mass by preventing $\beta$ cell replication (Table 1 ). The mutant transgene is not expressed in the exocrine compartment (25).

Figure 1 summarizes expression patterns of Insr and FoxO1 transgenes in the 3 mouse strains. We detected Insr in liver and brain, but not in muscle extracts from L1 mice. As negative controls, we show liver extracts from $\mathrm{Insr}^{-/}$mice and brain or muscle extracts from L2 transgenic knockout mice, which express Insr only in liver and $\beta$ cells (26) (Figure 1A). The mutant FoxO1 transgene was tagged with a c-Myc epitope to distinguish it from the endogenous protein (25). We detected expression of the c-Myc-FoxO1 fusion protein by immunoprecipitation with anti-c-Myc antiserum followed by immunoblotting with antiFoxO1 antiserum in liver of lines 307 and 305, but not in WT mice (Figure 1B). We also detected expression of c-Myc-FoxO1 fusion protein in $\beta$ cells of 305 mice, but not 307 or WT mice, by immunohistochemistry of pancreatic sections (25). It should be noted that the transthyretin-driven transgene is expressed in approximately $80 \%$ of all $\beta$ cells (Figure 1C).
Early-onset diabetes in double-mutant mice. Transgenic mice carrying the various mutations were born with the expected Mendelian frequency. At 2 weeks of age, all mutants showed an approximately $20 \%$ decrease in body weight compared with WT mice (Table 2). Metabolic analyses indicated that mean glucose levels of single and double transgenics did not differ from WT mice. However, approximately $14 \%$ of $\mathrm{L} 1 / 307$ and approximately $27 \%$ of $\mathrm{L} 1 / 305$ double-transgenic mice had glucose levels in the diabetic range (>200 mg/dl) (Figure 2A). L1 mice showed a 7-fold increase in insulin levels compared with WT mice (Figure 2B) (26). The 307 and 305 mice had insulin levels similar to WT mice. In contrast, both double mutants showed hyperinsulinemia. L1/307 had the same levels as L1 mice whereas L1/305 mice had approximately $55 \%$ lower levels than L1 mice. It should be noted that mean glucose levels and percentage of diabetic mice were higher in L1/305 than in L1/307 mice. Therefore, L1/305 mice have a more severe metabolic derangement than $\mathrm{L} 1 / 307$ mice. We also measured $\beta$ cell mass using immunohistochemistry on pancreatic sections. We failed to detect significant differences among the various genotypes (Figure 2C). However, since the mutant mice are growth retarded, one would predict that, in order to achieve the same $\beta$ cell mass as WT mice, $\beta$ cell replication rates should be increased. Indeed, when we measured proliferation as the percentage of Ki67positive $\beta$ cells, we detected an approximately 2 -fold increase in all mutant mice (Figure 2D).

At 8 weeks of age, both L1/307 and L1/305 mice had developed overt diabetes whereas 307 and 305 mice showed slight increases in glucose levels (25) (Figure 3A). Most of the double-mutant mice succumbed to diabetes between 4 and 12 months of age. Therefore, to avoid the confounding effects of chronic hyperglycemia, we carried out metabolic measurements in 8-week-old mice. L1 mice showed a 25 -fold increase in insulin levels with normal glucose levels, indicative of a state of compensated insulin resistance. L1/307 and L1/305 mice had greater than 50-fold higher insulin levels than WT mice, but this increase failed to prevent diabetes (Figure 3B). 


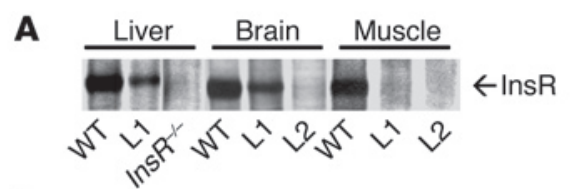

B

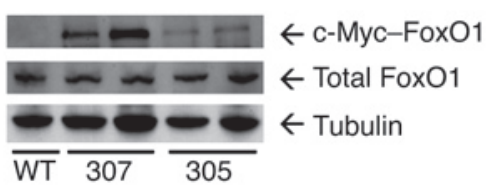

C
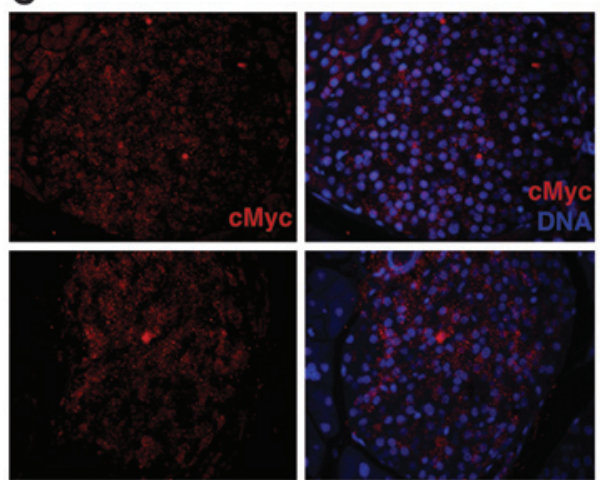

307
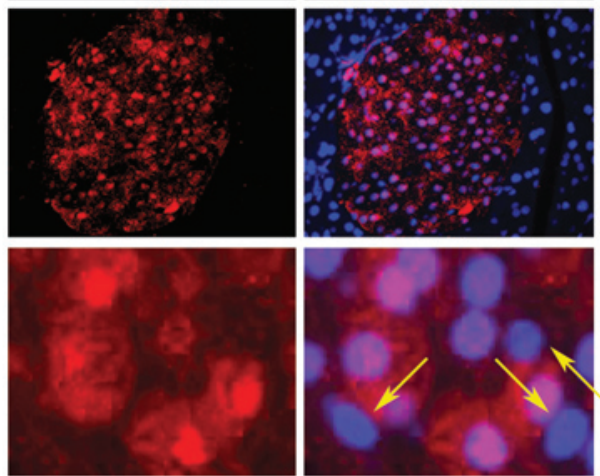

305

305

We have previously shown that $\mathrm{L} 1$ mice have normal hepatic insulin signaling and gene expression (26) while 307 and 305 mice have increased levels of gluconeogenic genes (25). Measurements of hepatic insulin signaling and gene expression in doublemutant mice L1/305 and L1/307 showed the expected metabolic abnormalities, with increased expression of glucogenetic enzymes (Pck1 and G6pc) accompanied by normal Insr content and phosphorylation (data not shown).

Expression of the FoxO1 transgene in $\beta$ cells prevents expansion of $\beta$ cell mass. We next examined islet morphology (Figure 4, A and B) and $\beta$ cell mass (Figure 4C) in 8-week-old mice. L1 mice showed a 2.4-fold increase in $\beta$ cell mass whereas neither 307 nor 305 transgenics showed significant differences compared with WT mice. L1/307 double transgenics showed a 3.4-fold increase in $\beta$ cell mass while $\mathrm{L} 1 / 305$ mice showed a $60 \%$ increase compared with WT but an approximately $65 \%$ decrease compared with single L1 transgenics (Figure 4C). These data are consistent with the hypothesis that hepatic expression of the FoxO1 transgene causes

\section{Figure 1}

Detection of c-Myc-FoxO1 fusion proteins. (A) Insr Western blot. We detected Insr by Western blotting of liver, brain, and muscle extracts of WT mice. L1 mice express Insr in liver and brain, but not muscle. Insr ${ }^{-1}$ mice do not express Insr in liver while L2 mice are Insr transgenic knockouts with Insr expression limited to liver and $\beta$ cells (26). (B) FoxO1 Western blot. We performed immunoprecipitations with anti-c-Myc antiserum and Western blotting with anti-FoxO1 antiserum (upper panel). As a control, we show immunoblotting with anti-FoxO1 (middle panel) and antitubulin antisera (lower panel). (C) Pancreatic immunohistochemistry. We performed immunostaining of pancreatic sections from WT, 307, and 305 mice with anti-c-Myc antiserum to detect transgene-encoded FoxO1 (left panels, red). DNA counterstaining with DAPI is shown in right panels. We show representative sections to illustrate the difference between transgene-positive and transgene-negative cells, indicated by the yellow arrows. Magnification, $\times 40$ (top 3 rows); $\times 100$ (bottom row).

diabetes by augmenting insulin resistance while $\beta$ cell expression of the FoxO1 transgene does so by curtailing expansion of $\beta$ cell mass. The relative increase of $\beta$ cell mass in $\mathrm{L} 1 / 305$ compared with WT or 305 mice (Figure 4C) can be accounted for by increased replication of the $20 \%$ of $\beta$ cells that do not express the FoxO1 transgene (Figure 1C).

To examine why $\beta$ cell mass failed to increase in L1/305 mice, we measured rates of $\beta$ cell proliferation and apoptosis. Only occasional apoptotic cells $(<0.001 \%$ of $\beta$ cells) were present in all mice analyzed, and we did not detect differences among the various genotypes. Proliferating $\beta$ cells, as measured by Ki67 incorporation, were rare in WT, $307, \mathrm{~L} 1$, and $\mathrm{L} 1 / 307$ mice ( $<0.01 \%$ of $\beta$ cells) but were completely absent in 305 and L1/305 mice, indicating that proliferation was effectively prevented by the FoxO1 transgene. It should be noted that, although L1/305 mice had smaller $\beta$ cell mass than $\mathrm{L} 1 / 307$ mice, their circulating insulin levels were similar, suggesting that they compensated for peripheral insulin resistance by increasing insulin secretion. These data indicate that, while the FoxO1 transgene inhibits $\beta$ cell proliferation, it does not adversely affect insulin secretion. Indeed, we have recently reported that FoxO1 promotes expression of the insulin gene transcription factors NeuroD and MafA (27).

Effects of the FoxO1 transgene on $\beta$ cell neogenesis. There is continuing controversy on the role of neogenesis versus proliferation in $\beta$ cell turnover (4). We explored this issue in our experimental crosses. The rationale was that, since the FoxO1 transgene is expressed from the transthyretin promoter, it should affect proliferation not only of terminally differentiated $\beta$ cells but also of duct cells, a site of transthyretin expression (28). We obtained 2 surrogate measures of $\beta$ cell "neogenesis" by counting $\beta$ cell singlets/doublets and $\beta$ cells abutting the lumen of pancreatic ducts $(4,21,22)$. While neither measure can be unequivocally assumed to represent neogenesis, certain testable predictions can be made.

If singlets/doublets arise from undifferentiated progenitors in response to insulin resistance, their number should increase in

\section{Table 2}

Body weights of 2-week-old mice

$\begin{array}{lccccc}\text { WT }(n=13) & \text { L1 }(n=10) & \mathbf{3 0 7}(n=10) & \mathbf{3 0 5}(n=8) & \text { L1/307 }(n=7) & \text { L1/305 }(n=11) \\ 9.3 \pm 0.5 & 7.4 \pm 0.8 & 6.1 \pm 0.5^{\mathrm{B}} & 7.7 \pm 0.4^{\mathrm{A}} & 7.4 \pm 0.4^{\mathrm{A}} & 7.3 \pm 0.4^{\mathrm{A}}\end{array}$

Weights are expressed in grams. ${ }^{A} P<0.01$ versus $W T$. ${ }^{B} P<0.01$ versus $W T, 305, L 1 / 305$, and L1/307. 
A

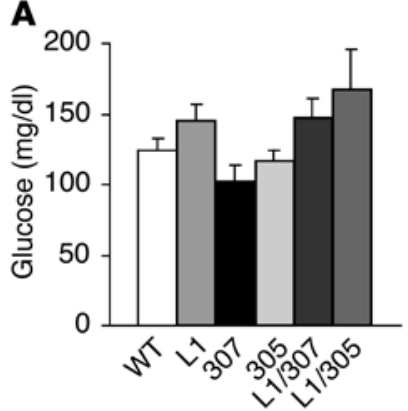

C

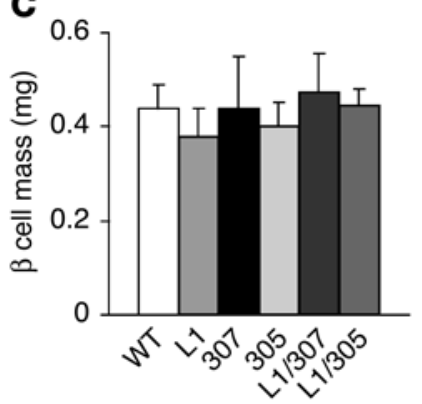

B

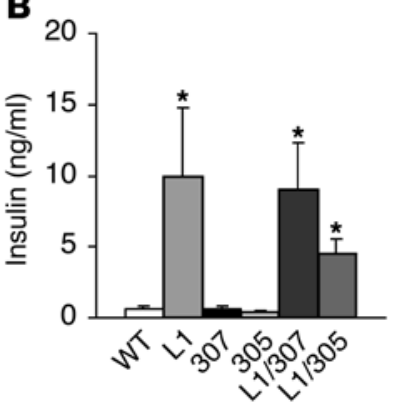

D

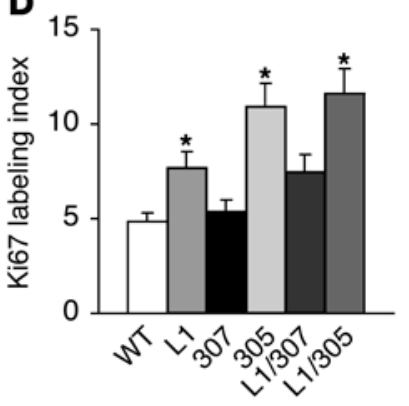

L1/305 transgenics to compensate for the failure to increase $\beta$ cell mass through replication because the FoxO1 transgene is not expressed in progenitor cells. If they arise from replication, their number should decrease in $\mathrm{L} 1 / 305$ transgenics in proportion to the overall decrease in $\beta$ cell mass because the FoxO1 transgene prevents their replication. Indeed, the number of singlets/doublets remained constant throughout the various genotypes, except in L1/305 double mutants. The latter showed a $65 \%$ decrease compared with WT mice and a 55\% decrease compared with L1 mice (Figure 5A). These data are consistent with the notion that $\beta$ cell singlets/doublets arise from replication of existing $\beta$ cells (4).

With respect to the ductal origin of $\beta$ cells, the prediction was that, if duct epithelia are a site of $\beta$ cell neogenesis, the number of periductal $\beta$ cells should decrease in mice expressing the FoxO1 transgene, as the ductal promoter-driven transgene will curtail expansion of this cell population. If $\beta$ cells arise from other cell types, residing by happenstance in proximity to ducts, their number should increase to compensate for peripheral insulin resistance. In $\mathrm{L} 1$ mice, despite the increase in $\beta$ cell mass, we failed to detect an increase in the number of duct-associated $\beta$ cells. Unexpectedly, we saw a decrease in 307 mice, which, however, do not express the mutant transgene in ducts (25). The 305 transgenics had numbers similar to those of WT and L1 mice. Both L1/307 and L1/305 transgenics showed a decrease in periductal $\beta$ cells compared with L1 mice (Figure 5B). As a control, we measured overall proliferation rates in pancreatic ducts and detected no differences among the various genotypes (Figure 5C). The decrease of periductal $\beta$ cells in L1/305 could be construed as consistent with the ductal cell hypothesis. However, because a similar decrease was present in L1/307 mice, which do not express the transgene in ducts, this finding cannot be attributed solely to the effects of the FoxO1 transgene. The conclusion of these experiments is that the reduction of $\beta$ cell mass in L1/305 transgenics is largely, if not exclusively, due to impaired $\beta$ cell replication in adult mice. If there is a contribution from extra-

\section{Figure 2}

Metabolic data in 2-week-old mice. We measured glucose $(\mathbf{A})$ and insulin levels (B) in random-fed mice. (C) $\beta$ cell mass. We determined the percentage of the area of pancreas occupied by $\beta$ cells on pancreatic sections stained with anti-insulin antiserum. We scored at least 3 animals per genotype and 4 sections per animal spaced $80 \mu \mathrm{m}$ apart. We then determined $\beta$ cell mass by multiplying the area for pancreatic weight. (D) We measured the percentage of Ki67-positive cells (Ki67-labeling index) in 3 sections for each of 3 animals per genotype. ${ }^{*} P<0.05$ versus WT.

islet $\beta$ cells (ductal or otherwise), it is quantitatively small. Our data also caution against a quantitative interpretation of periductal $\beta$ cells until more specific lineage markers are employed to address this question.

A paracrine mechanism of $\beta$ cell expansion. The failure to increase $\beta$ cell mass in L1/305 transgenics may be due to the complex genetic makeup of these mice, which lack Insr in many cell types (26). To obtain independent evidence for the role of $\beta$ cell proliferation in insulin-resistant states, we developed a model of $\beta$ cell hyperplasia by ectopic growth factor expression in the pancreas. To this end, we generated elastase-IGF2 transgenic mice (henceforth, El-IGF2) (29). We chose IGF2 because it binds with equal affinity to the 2 receptors of the insulin/IGF family, Insr and Igf1 receptor (30). Moreover, since IGF2 is not expressed in adult rodents (31), changes in IGF2 levels are readily detectable. The elastase promoter was chosen because it is expressed in exocrine pancreas and ducts, thus enabling transgenically produced IGF 2 to act in a paracrine fashion on $\beta$ cells in view of the anatomical contiguity of the exocrine/duct and endocrine compartments (32). El-IGF2 transgenic mice showed elevated IGF2 levels in whole pancreas extracts (Figure 6, A and B) without detectable circulating IGF2 in plasma (data not shown). Four-week-old El-IGF2 mice had a slight decrease in glucose levels and a 2-fold increase in insulin levels, associated with a 2-fold increase in Ki67 labeling of $\beta$ cells (Figure 6, C-E). Total pancreas weight (Figure 6F) and $\beta$ cell mass (Figure $6 \mathrm{G}$ ) were similar to WT mice at this age. In age-matched El-IGF2/305 mice, we observed whole-pancreas IGF2 (Figure 6, A and B), plasma glucose, and insulin levels similar to those of El-IGF2 mice (Figure 6, C and D). In contrast, we detected trends toward decreased Ki67-positive $\beta$ cells (Figure 6E) and $\beta$ cell mass (Figure $6 \mathrm{G}$ ), with normal pancreas weight (Figure $6 \mathrm{~F}$ ). Because the El-IGF2/305 mice do not develop diabetes, we could
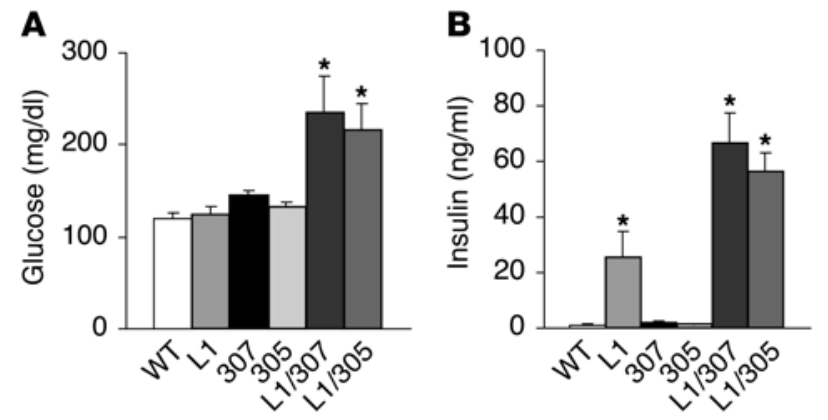

\section{Figure 3}

Metabolic data in 2-month-old mice. We measured glucose $(\mathbf{A})$ and insulin levels $(\mathbf{B})$ in random-fed 2 -month-old mice. ${ }^{*} P<0.01$ versus WT, 305, and 307. 
A

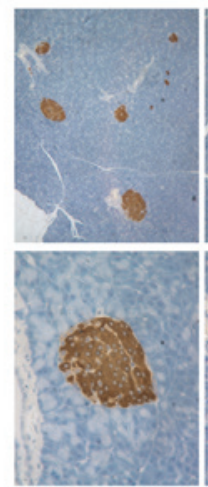

WT

B

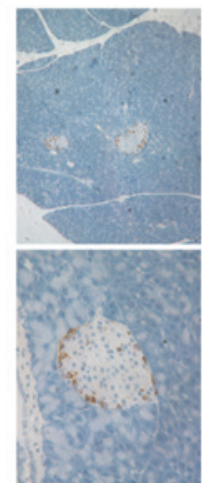

WT

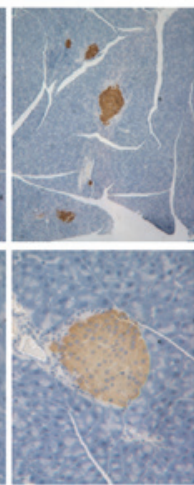

305

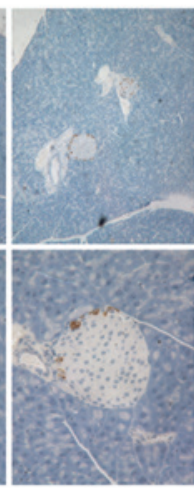

305

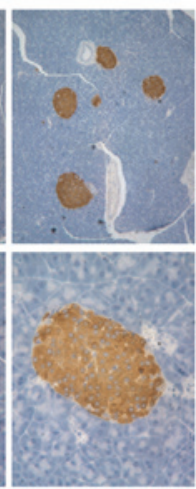

307

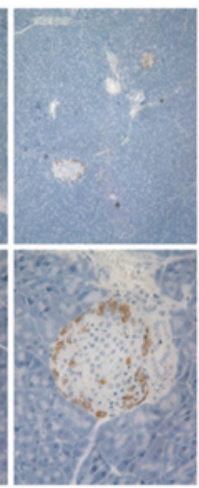

307

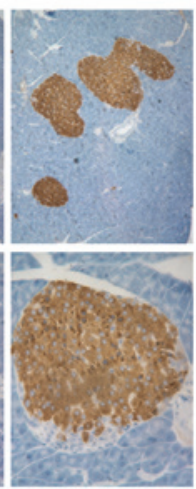

L1

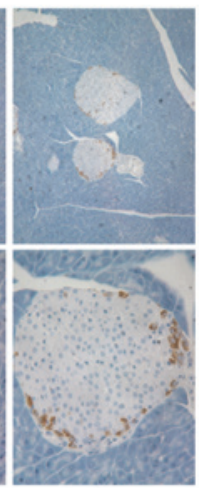

L1

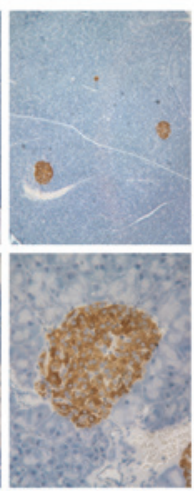

$\mathrm{L} 1 \times 305$
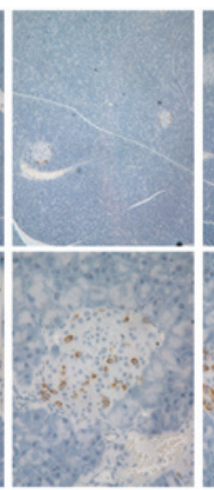

$\mathrm{L} 1 \times 305$

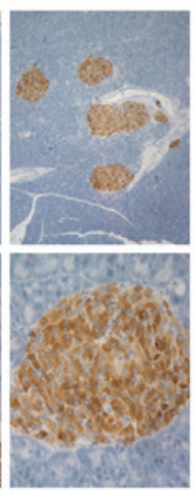

$\mathrm{L} 1 \times 307$
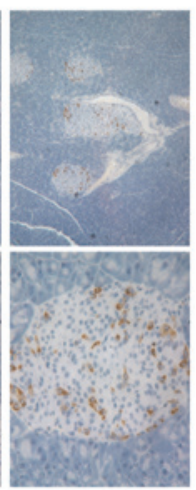

L1 $\times 307$

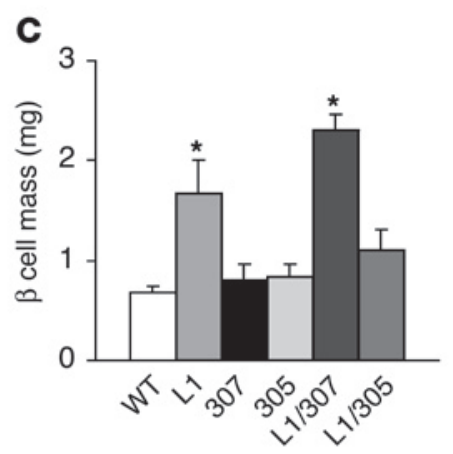

Figure 4

Pancreatic histology and morphometry. (A) Insulin immunohistochemistry. (B) Glucagon immunohistochemistry. Magnification, $\times 10$ (top panels); $\times 40$ (bottom panels). (C) Measurements of $\beta$ cell mass. We determined $\beta$ cell mass as indicated in Methods, following immunohistochemistry with antiinsulin antiserum. We scored at least 3 animals per genotype and 4 sections per animal spaced $80 \mu \mathrm{m}$ apart. ${ }^{*} P<0.01$ versus WT, 305 , and 307.

also measure the long-term effects of the FoxO1 transgene in 6-month-old animals (Figure 7). At this age, we observed normal glucose levels across all genotypes and only modest increases of insulin levels ( $<2$-fold) in all transgenics. $\beta$ cell mass increased approximately 2-fold in El-IGF2 mice but decreased approximately $30 \%$ in 305 mice and approximately $40 \%$ in El-IGF2/305 double-transgenic mice.

\section{Discussion}

The goal of this work was to analyze the mechanism(s) of $\beta$ cell hyperplasia in insulin-resistant states. To this end, we generated transgenic models of combined insulin resistance and $\beta$ cell failure by introducing mutations that affect insulin signaling in peripheral tissues and $\beta$ cells of mice. Our findings indicate that expansion of $\beta$ cell mass in response to insulin resistance requires FoxO1 nuclear exclusion in $\beta$ cells and is therefore primarily a mitotic response of terminally differentiated cells, as opposed to de novo differentiation of progenitor cells. In addition, we show that $\beta$ cell hyperplasia can occur in response to locally acting growth factors via a paracrine mechanism. The present data cast FoxO1 as a key mediator of $\beta$ cell proliferation, but we should note that nuclear exclusion of FoxO1 is necessary but not sufficient for $\beta$ cell replication. In fact, FoxO1 can be physiologically detected in the cytoplasm of nondividing $\beta$ cells (23), indicating that additional triggers are required to promote $\beta$ cell mitosis. Similarly, it should be emphasized that the role of the FoxO1 transgene to prevent expansion $\beta$ cell mass appears to reflect an effect on $\beta$ cell turnover in adult mice and not during early life, when $\beta$ cell-proliferation rates are unaffected by the mutant FoxO1 protein. This observation could be due to late onset of transgene expression or to compensatory hyperproliferation of the transgene-negative $\beta$ cell subpopulation, potentially reflecting the first wave of postnatal $\beta$ cell growth described in rodents (33). Nonetheless, when these observations are viewed in the context of prior work showing that FoxO1 haploinsufficiency partially restores $\beta$ cell proliferation in Irs2 knockout mice (23), they begin to outline an important role for FoxO1 in $\beta$ cell turnover.

The relationship between insulin sensitivity and $\beta$ cell compensation has been the object of extensive investigation. Data in humans and rodents indicate that there exists a negative correlation between insulin sensitivity and insulin secretion (13). But why does increased insulin secretion cause an expansion of $\beta$ cell mass? There are several mutually compatible possibilities. The $\beta$ cell may possess a sensing mechanism to promote mitosis if insulin secretion exceeds a certain threshold, or insulin resistance could promote the generation of humoral mediators of $\beta$ cell proliferation (34). A less circuitous explanation is that secreted insulin acts as a $\beta$ cell proliferation factor (13). This is consistent with the observation that disruption of insulin signaling, for example, in Irs 2 knockouts, affects $\beta$ cell mass $(18,19)$. 

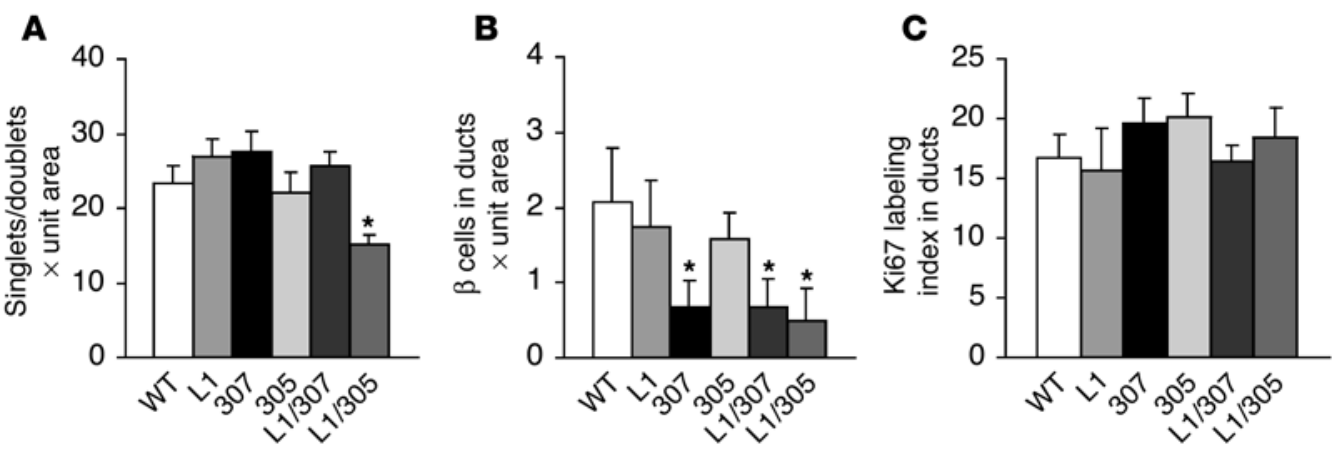

\section{Figure 5}

Measurements of $\beta$ cell singlets/doublets and ductal $\beta$ cells. (A) We measured the number of extra-islet $\beta$ cell singlets and doublets and normalized the number by the area of pancreas examined. At least 4 independent sections were scored for each of 3 animals of each genotype. ${ }^{*} P<0.01$ versus WT. (B) For measurements of duct-associated $\beta$ cells, we scored insulin-positive cells that could be unequivocally located within the lumen of a pancreatic duct and were not part of an islet. The number was normalized by section area. At least 4 independent sections were scored for each of 3 animals of each genotype. ${ }^{\star} P<0.01$ versus WT, 305, and 307. (C) Duct cell proliferation. We determined the Ki67-labeling index of duct cells, identified based on their anatomical location. We scored 6 mice per genotype and 3 sections for each animal.

It has been argued that this mechanism cannot explain the human islet's adaptation to insulin resistance because individuals with mutations that affect insulin signaling (for example, patients with Donohue syndrome, or leprechaunism, a rare syndrome caused by INSR mutations) have exceedingly high plasma insulin levels (35). However, it should be noted that insulin at high concentrations can activate the cognate IGF1 receptor, and we have shown that the 2 receptors play overlapping roles in organismal growth $(36,37)$. Therefore, islet compensation could be mediated by IGF1 receptors, as are other side effects of extreme insulin resistance in humans (35). Regardless of whether human and rodent islets respond in similar or diverging fashions to insulin resistance, the data in IGF2 transgenic mice provide proof of principle that $\beta$ cell hyperplasia could occur as a response to an alteration of the local environment, in the absence of systemic metabolic changes.

From where do $\beta$ cells arise in adult life? Lineage tracing studies, using the insulin promoter (4), and the present work, using the transthyretin promoter, support the view that $\beta$ cell mass is controlled via replication rather than de novo differentiation. A similar conclusion is supported by studies of physiologic islet turnover in cyclin D (38) and E2F1 knockouts $(39,40)$, both of which affect cell-cycle progression and are in the FoxO1 pathway (41-43). When compared with these prior findings, there are 3 aspects to the present work that we believe are new. First, we have studied $\beta$ cell hyperplasia associated with insulin-resistant states, as opposed to physiologic $\beta$ cell turnover. Second, unlike most other studies in this field, which have used the Insulin2 promoter to drive expression of reporter genes, we have used the transthyretin promoter to provide independent evidence that replication is a feature of terminally differentiated $\beta$ cells. Finally, the use of the transthyretin promoter to drive FoxO1 expression has also enabled us to address whether there is a contribution from transthyretin-expressing duct epithelia to the process of $\beta$ cell compensation to insulin resistance. The data in this respect are equivocal since a decrease in periductal $\beta$ cells is observed both in L1/305 mice and L1/307 mice, which do not express the FoxO 1 transgene in ductal cells and have otherwise robust $\beta$ cell compensation. Our data do not rule out the presence of progenitor endocrine cells in the postnatal pancreas, nor do they imply that these cells are unimportant, since even a rare population could potentially be expanded in vitro to produce a permanent source of $\beta$ cells for transplantation. Indeed, the identification of these endocrine progenitors remains an important goal of diabetes research.

\section{Methods}

Animal production and phenotypic analysis. The Columbia University Institutional Animal Care and Utilization Committee approved all procedures. We have described L1 (26), 307, and 305 mice (25) in previous publications. The animals were maintained on a mixed background derived from 129/sv, C57BL/6J, and FVB mice. We measured glucose and insulin as described previously (44). We genotyped animals using PCR on tail DNA as described $(25,26)$. To generate transgenic mice expressing IGF2 in the exocrine pancreas (IGF2), we cloned the IGF2 cDNA downstream from the 500-bp human Elastase promoter (29). We identified transgenic mice using PCR genotyping with the following primers: forward, 5'-TAACTGAGTGCCGGCCTTGTTCTG-3' (sequence from the elastase promoter); and reverse, 5'-TGAGAAGCACCAACATCGACTTCC-3' (sequence from Igf2 cDNA). These primers amplify a 279-bp product.

Western analysis. We prepared nuclear extracts from liver as described previously (25). We immunoprecipitated equal amounts of liver extracts with antibody against c-Myc (9E10; Santa Cruz Biotechnology Inc.), resolved the immunoprecipitants on $8 \%$ SDS-PAGE, and transferred to nitrocellulose membrane (Protoran; Schleicher \& Schuell BioScience Inc.). We probed the membranes with anti-FoxO1 antibody (H128; Santa Cruz Biotechnology Inc.) at a 1:200 dilution, followed by detection with horseradish peroxidase-coupled anti-rabbit IgG (Amersham Pharmacia Biotech) at a 1:5,000 dilution using the ECL detection system (Amersham Pharmacia Biotech). For detection of endogenous and transgene-encoded FoxO1, we resolved equal amounts of liver extracts by $8 \%$ SDS-PAGE and transferred them to nitrocellulose membranes. We detected FoxO1 proteins as described above. To normalize for protein content, we stripped the filters and reprobed them with anti- $\beta$-tubulin antibody (TU27; CRP Inc.) at a 1:1,000 dilution, followed by detection with anti-mouse IgG antiserum (Amersham Pharmacia Biotech) and ECL.

Immunohistochemical analysis of pancreatic islets. We isolated and weighed pancreata and then fixed them overnight in $2 \%$ paraformaldehyde solu- 

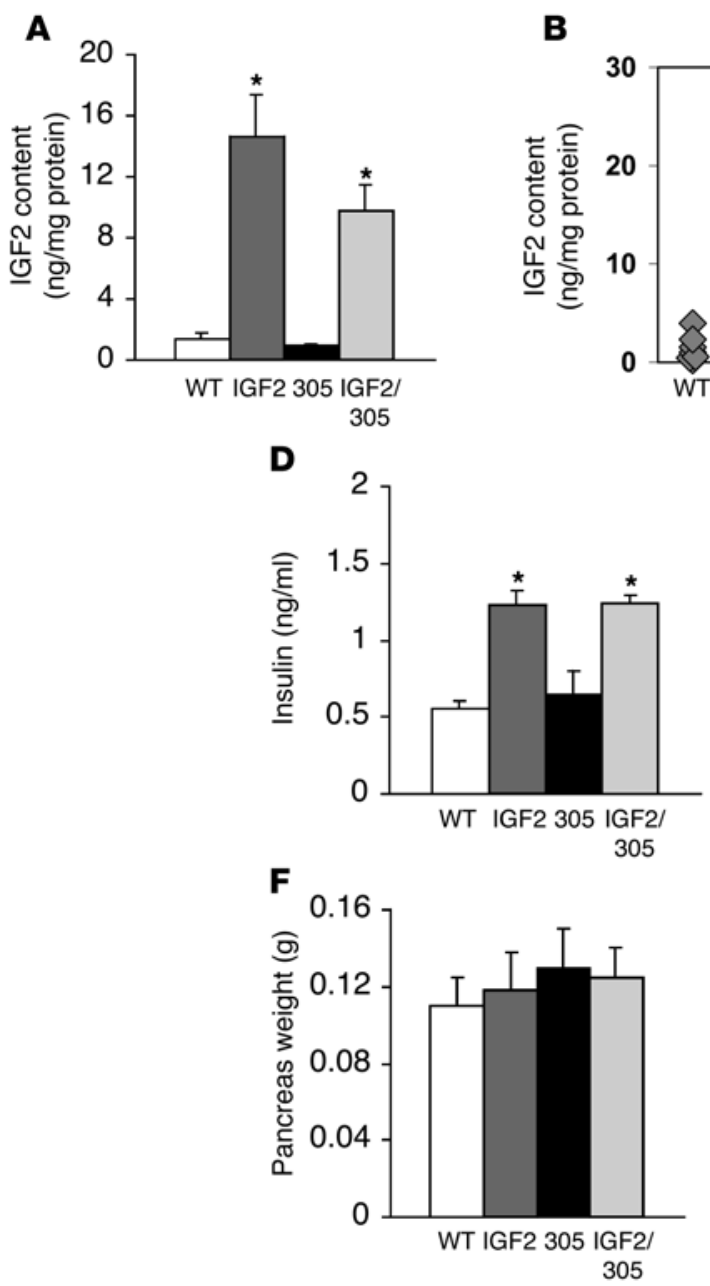

B

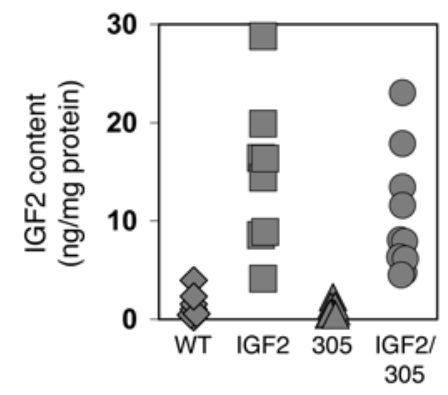

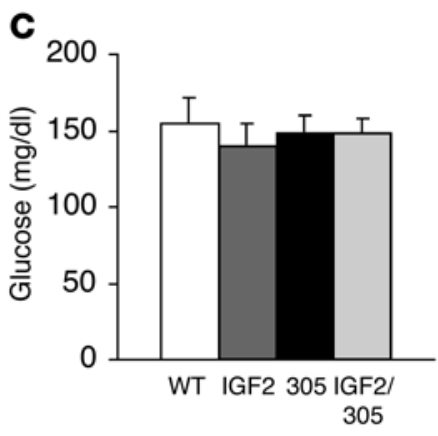

\section{E}
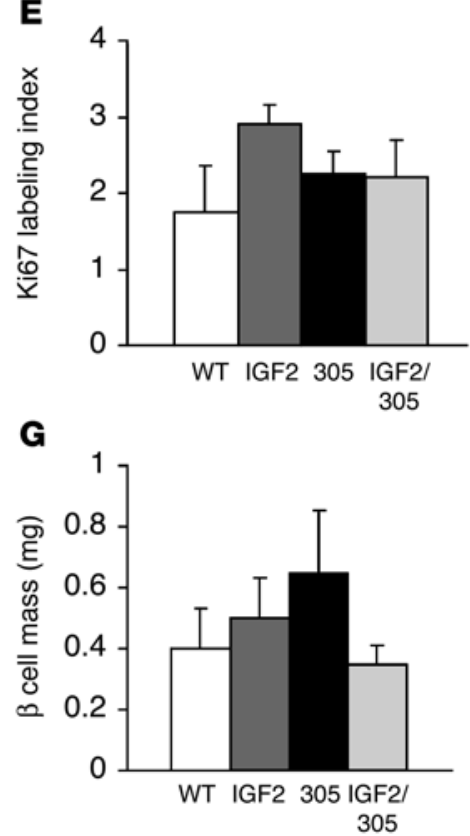

Figure 6

IGF2 levels and metabolic data in 4-week-old mice. (A) We measured mean \pm SEM IGF2 levels using an ELISA assay in acid-ethanol extracts from whole pancreata. ${ }^{\star} P<0.01$ versus WT and 305. (B) IGF2 levels in individual transgenic mice and WT controls. (C) Glucose and (D) insulin levels in random-fed mice. (E) $\beta$ cell replication. We determined the labeling index of pancreatic $\beta$ cells by double immunohistochemistry with antiKi67 and anti-insulin antibodies. We scored at least 3 animals per genotype and 4 sections per animal. (F) Pancreas weight in El-IGF2, 305, and WT mice in 6 mice for each genotype. (G) We determined $\beta$ cell mass as indicated in Methods in 6 mice for each genotype. IGF2, El-IGF2.

tion, embedded them in paraffin, and obtained $4-\mu \mathrm{m}$-thick sections. We immunostained sections for $\beta$ and $\alpha$ cells using mouse anti-insulin and anti-glucagon antibodies, respectively (Sigma-Aldrich). To detect transgene-expressing $\beta$ cells, we immunostained sections using anti-c-Myc antibody (2276; Cell Signaling Technology). We detected the antiserum using rhodamine- and fluorescein-conjugated secondary antibodies (Jackson ImmunoResearch Laboratories Inc.).

Figure 7

Metabolic data in 6-month-old mice. We measured glucose (A) and insulin levels $(\mathbf{B})$ in random-fed mice. (C) Pancreatic morphometry. We determined $\beta$ cell mass as indicated in Methods. We scored at least 3 animals per genotype and 4 sections per animal. ${ }^{*} P<0.05$ versus WT.

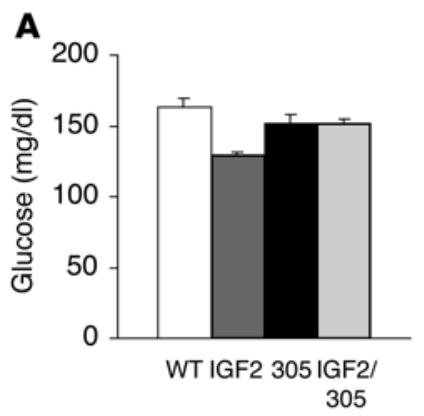

Pancreatic morphometry. We analyzed 3 sections, spaced at least $80 \mu \mathrm{m}$ apart, of each animal using SPOT software (version 4.0.8; Diagnostic Instruments) and Image-Pro Plus software (version 5.0.1; MediaCybernetics Inc.). We studied 4 to 6 animals of each genotype for each age investigated. We calculated $\beta$ cell mass by multiplying the percentage of surveyed pancreatic area occupied by $\beta$ cells by total pancreatic weight. We determined the $\beta$ cell Ki67-labeling index by dividing the number of Ki67-positive by the total number of $\beta$ cells in at least 3

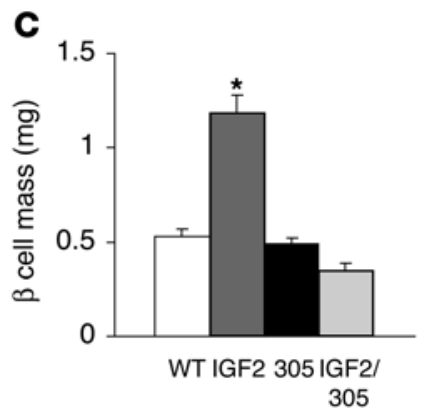


separate sections in 3 to 6 animals for each genotype (16). For duct proliferation analysis, we performed a similar calculation in anatomically identifiable ducts.

Measurement of IGF2 content. We prepared pancreatic extracts in $12.5 \%$ $2 \mathrm{~N} \mathrm{HCl}, 87.5 \%$ ethanol at room temperature for 30 minutes. After centrifugation, the supernatant was neutralized by addition of $\mathrm{NaOH}$. We measured IGF2 content by ELISA using the mouse IGF-II DuoSet (R\&D Systems) and total protein by BCA protein assay (Pierce Biotechnology) according to the manufacturers' protocols.

Statistics. We calculated descriptive statistics and ANOVA followed by Fisher's test using the Statsview software (SAS Institute Inc.).

\section{Acknowledgments}

This work was supported by NIH grants DK64819 and DK63608 (Columbia Diabetes and Endocrinology Research Center), the Berrie Program in Cell-Based Therapies at Columbia Univer- sity, and Medical Research Council UK grant G9810961. We thank Qun Xu and Qiong Li for skilled technical assistance with immunohistochemistry and members of the Accili laboratory for stimulating discussions.

Received for publication March 7, 2005, and accepted in revised form December 22, 2005

Address correspondence to: Domenico Accili, Berrie Research Pavilion, 1150 St. Nicholas Avenue, Room 238A, New York, New York 10032, USA. Phone: (212) 851-5332; Fax: (212) 851-5331; E-mail: da230@columbia.edu.

Haruka Okamoto and Marta Letizia Hribal contributed equally to this work.
1. Bell, G.I., and Polonsky, K.S. 2001. Diabetes mellitus and genetically programmed defects in beta-cell function. Nature. 414:788-791.

2. Gradwohl, G., Dierich, A., LeMeur, M., and Guillemot, F. 2000. Neurogenin3 is required for the development of the 4 endocrine cell lineages of the pancreas. Proc. Natl. Acad. Sci. U. S. A. 97:1607-1611.

3. Schwitzgebel, V.M., et al. 2000. Expression of neurogenin3 reveals an islet cell precursor population in the pancreas. Development. 127:3533-3542.

4. Dor, Y., Brown, J., Martinez, O.I., and Melton, D.A. 2004. Adult pancreatic beta-cells are formed by selfduplication rather than stem-cell differentiation. Nature. 429:41-46.

5. Bonner-Weir, S. 2000. Perspective: postnatal pancreatic beta cell growth. Endocrinology. 141:1926-1929.

6. Bouwens, L., and Pipeleers, D.G. 1998. Extra-insular beta cells associated with ductules are frequent in adult human pancreas. Diabetologia. 41:629-633.

7. Gu, G., Dubauskaite, J., and Melton, D.A. 2002. Direct evidence for the pancreatic lineage: NGN3+ cells are islet progenitors and are distinct from duct progenitors. Development. 129:2447-2457.

8. Vasavada, R.C., et al. 2000. Targeted expression of placental lactogen in the beta cells of transgenic mice results in beta cell proliferation, islet mass augmentation, and hypoglycemia. J. Biol. Chem. 275:15399-15406.

9. Garcia-Ocana, A., et al. 2000. Hepatocyte growth factor overexpression in the islet of transgenic mice increases beta cell proliferation, enhances islet mass, and induces mild hypoglycemia. J. Biol. Chem. 275:1226-1232.

10. George, M., et al. 2002. $\beta$ cell expression of IGF-I leads to recovery from type 1 diabetes. J. Clin. Invest. 109:1153-1163. doi:10.1172/JCI200212969.

11. Cousin, S.P., et al. 1999. Stimulation of pancreatic beta-cell proliferation by growth hormone is glucose-dependent: signal transduction via Janus kinase 2 (JAK2)/signal transducer and activator of transcription 5 (STAT5) with no crosstalk to insulin receptor substrate-mediated mitogenic signalling. Biochem. J. 344:649-658.

12. Milburn, J.L., Jr., et al. 1995. Pancreatic beta-cells in obesity. Evidence for induction of functional, morphologic, and metabolic abnormalities by increased long chain fatty acids. J. Biol. Chem. 270:1295-1299.

13. Accili, D. 2004. Lilly lecture 2003: the struggle for mastery in insulin action: from triumvirate to republic. Diabetes. 53:1633-1642.

14. Kulkarni, R.N., et al. 1999. Tissue-specific knockout of the insulin receptor in pancreatic beta cells creates an insulin secretory defect similar to that in type 2 diabetes. Cell. 96:329-339.

15. Kulkarni, R.N., et al. 2002. Beta-cell-specific deletion of the Igf1 receptor leads to hyperinsulinemia and glucose intolerance but does not alter beta-cell mass. Nat. Genet. 31:111-115.

16. Xuan, S., et al. 2002. Defective insulin secretion in pancreatic $\beta$ cells lacking type 1 IGF receptor. J. Clin. Invest. 110:1011-1019. doi:10.1172/ JCI200215276.

17. Kulkarni, R.N., et al. 1999. Altered function of insulin receptor substrate-1-deficient mouse islets and cultured $\beta$-cell lines. J. Clin. Invest. 104:R69-R75.

18. Withers, D.J., et al. 1998. Disruption of IRS-2 causes type 2 diabetes in mice. Nature. 391:900-904.

19. Kubota, N., et al. 2000. Disruption of insulin receptor substrate 2 causes type 2 diabetes because of liver insulin resistance and lack of compensatory beta-cell hyperplasia. Diabetes. 49:1880-1889.

20. Eto, K., et al. 2002. Phosphatidylinositol 3-kinase suppresses glucose-stimulated insulin secretion by affecting post-cytosolic $[\mathrm{Ca}(2+)]$ elevation signals. Diabetes. 51:87-97.

21. Bernal-Mizrachi, E., Wen, W., Stahlhut, S., Welling, C., and Permutt, M. 2001. Transgenic mice expressing a constitutively active $\mathrm{Akt} 1 / \mathrm{PKB} \alpha$ in pancreatic islet $\beta$-cells exhibit striking hypertrophy, hyperplasia, and hyperinsulinemia. J. Clin. Invest. 108:1631-1638. doi:10.1172/JCI200113785.

22. Tuttle, R.L., et al. 2001. Regulation of pancreatic beta-cell growth and survival by the serine/threonine protein kinase Akt1/PKBalpha. Nat. Med. 7:1133-1137

23. Kitamura, T., et al. 2002. The forkhead transcription factor FoxO1 links insulin signaling to Pdx1 regulation of pancreatic $\beta$ cell growth. J. Clin. Invest. 110:1839-1847. doi:10.1172/JCI200216857.

24. Johnson, J.D., et al. 2003. Increased islet apoptosis in Pdx1+/- mice. J. Clin. Invest. 111:1147-1160. doi:10.1172/JCI200316537.

25. Nakae, J., et al. 2002. Regulation of insulin action and pancreatic beta-cell function by mutated alleles of the gene encoding forkhead transcription factor FoxO1. Nat. Genet. 32:245-253.

26. Okamoto, H., et al. 2004. Transgenic rescue of insulin receptor-deficient mice. J. Clin. Invest. 114:214-223. doi:10.1172/JCI200421645.

27. Kitamura, Y.I., et al. 2005. FoxO1 protects against pancreatic beta cell failure through NeuroD and MafA induction. Cell Metab. 2:153-163.

28. Kato, M., Kato, K., Blaner, W.S., Chertow, B.S., and Goodman, D.S. 1985. Plasma and cellular retinoidbinding proteins and transthyretin (prealbumin) are all localized in the islets of Langerhans in the rat. Proc. Natl. Acad. Sci. U. S. A. 82:2488-2492.

29. Heller, R.S., et al. 2001. Improved glucose tolerance and acinar dysmorphogenesis by targeted expression of transcription factor PDX-1 to the exocrine pancreas. Diabetes. 50:1553-1561.

30. Frasca, F., et al. 1999. Insulin receptor isoform A, a newly recognized, high-affinity insulin- like growth factor II receptor in fetal and cancer cells. Mol. Cell. Biol. 19:3278-3288.

31. Nakae, J., Kido, Y., and Accili, D. 2001. Distinct and overlapping functions of insulin and IGF-I receptors. Endocr. Rev. 22:818-835.

32. Bertelli, E., Regoli, M., Orazioli, D., and Bendayan, M. 2001. Association between islets of Langerhans and pancreatic ductal system in adult rat. Where endocrine and exocrine meet together? Diabetologia. 44:575-584.

33. Finegood, D.T., Scaglia, L., and Bonner-Weir, S. 1995. Dynamics of beta-cell mass in the growing rat pancreas. Estimation with a simple mathematical model. Diabetes. 44:249-256.

34. Flier, S.N., Kulkarni, R.N., and Kahn, C.R. 2001. Evidence for a circulating islet cell growth factor in insulin- resistant states. Proc. Natl. Acad. Sci. U. S. A. 98:7475-7480.

35. Taylor, S.I. 1992. Lilly Lecture: molecular mechanisms of insulin resistance. Lessons from patients with mutations in the insulin-receptor gene. Diabetes. 41:1473-1490.

36. Kido, Y., et al. 2002. Effects of mutations in the insulin-like growth factor signaling system on embryonic pancreas development and beta-cell compensation to insulin resistance. J. Biol. Chem. 277:36740-36747.

37. Louvi, A., Accili, D., and Efstratiadis, A. 1997. Growthpromoting interaction of IGF-II with the insulin receptor during mouse embryonic development. Dev. Biol. 189:33-48.

38. Georgia, S., and Bhushan, A. 2004. $\beta$ cell replication is the primary mechanism for maintaining postnatal $\beta$ cell mass. J. Clin. Invest. 114:963-968. doi:10.1172/JCI200422098.

39. Fajas, L., et al. 2004. Impaired pancreatic growth, $\beta$ cell mass, and $\beta$ cell function in E2F1 (-/-) mice. J. Clin. Invest. 113:1288-1295. doi:10.1172/ JCI200418555.

40. Li, F.X., et al. 2003. The development of diabetes in E2f1/E2f2 mutant mice reveals important roles for bone marrow-derived cells in preventing islet cell loss. Proc. Natl. Acad. Sci. U. S. A. 100:12935-12940.

41. Schmidt, M., et al. 2002. Cell cycle inhibition by FoxO forkhead transcription factors involves downregulation of cyclin D. Mol. Cell. Biol. 22:7842-7852.

42. Fernandez de Mattos, S., et al. 2004. FoxO3a and BCR-ABL regulate cyclin D2 transcription through a STAT5/BCL6-dependent mechanism. Mol. Cell. Biol. 24:10058-10071.

43. Nakae, J., et al. 2003. The forkhead transcription factor foxo1 regulates adipocyte differentiation. Dev. Cell. 4:119-129.

44. Kido, Y., et al. 2000. Tissue-specific insulin resistance in mice with mutations in the insulin receptor, IRS-1, and IRS-2. J. Clin. Invest. 105:199-205. 